\title{
Transcriptional Factor NF- $\kappa$ B as a Target for Therapy in Parkinson's Disease
}

\author{
Patrick M. Flood, ${ }^{1}$ Li Qian, ${ }^{1}$ Lynda J. Peterson, ${ }^{1}$ Feng Zhang, ${ }^{2,3}$ Jing-Shan Shi, ${ }^{2}$ \\ Hui-Ming Gao, ${ }^{3}$ and Jau-Shyong Hong ${ }^{3}$ \\ ${ }^{1}$ Department of Periodontology and the Comprehensive Center for Inflammatory Disorders, University of North Carolina, Chapel Hill, \\ NC 27599-7454, USA \\ ${ }^{2}$ Department of Pharmacology and Key Lab of Pharmacology of Guizhou, Zunji Medical College, Zunji, China \\ ${ }^{3}$ Neuropharmacology Section, Laboratory of Toxicology and Pharmacology, National Institute of Environmental Health Sciences, \\ National Institutes of Health, Research Triangle Park, NC 27709, USA
}

Correspondence should be addressed to Patrick M. Flood, pat_flood@dentistry.unc.edu

Received 10 November 2010; Accepted 21 February 2011

Academic Editor: Fernando Pitossi

Copyright (C) 2011 Patrick M. Flood et al. This is an open access article distributed under the Creative Commons Attribution License, which permits unrestricted use, distribution, and reproduction in any medium, provided the original work is properly cited.

\begin{abstract}
Parkinson's disease (PD) is a neurodegenerative condition characterized by chronic inflammation. Nuclear factor $\kappa \mathrm{B}(\mathrm{NF}-\kappa \mathrm{B})$ is a family of inducible transcription factors that are expressed in a wide variety of cells and tissues, including microglia, astrocytes, and neurons, and the classical NF- $\kappa \mathrm{B}$ pathway plays a key role in the activation and regulation of inflammatory mediator production during inflammation. Activation of the classical NF- $\kappa \mathrm{B}$ pathway is mediated through the activity of the IKK kinase complex, which consists of a heterotrimer of IKK $\alpha$, IKK $\beta$, and IKK $\gamma$ subunits. Targeting NF- $\kappa$ B has been proposed as an approach to the treatment of acute and chronic inflammatory conditions, and the use of inhibitors specific for either IKK $\beta$ or IKK $\gamma$ has now been found to inhibit neurodegeneration of TH+ DA-producing neurons in murine and primate models of Parkinson's disease. These studies suggest that targeting the classical pathway of NF- $\kappa$ B through the inhibition of the IKK complex can serve as a useful therapeutic approach to the treatment of PD.
\end{abstract}

\section{Introduction}

Parkinson's disease (PD) is a progressive degenerative disorder of the central nervous system (CNS) that leads to impairment of motor skills and speech, as well as other functions. While the disease mechanisms that ultimately cause PD are still unclear, it is believed that the progressive nature of $\mathrm{PD}$ is characterized by chronic inflammationinduced neurodegeneration of dopamine-producing neurons within the substantia nigra $(\mathrm{SN})$ and striatum [1-4]. It is now well documented that microglial activation results in the loss of dopaminergic neurons (DA-neurons). The premise of microglia activation in PD has been supported by analysis of postmortem brains from PD patients, which provides clear evidence of microglia activation in the SN. In the brains of patients with $\mathrm{PD}$, large numbers of human leukocyte antigen (HLA-DR) and CD11b-positive reactive microglia were found in the $\mathrm{SN}$, a region in which the degeneration of DA-neurons was most prominent [5-8]. In addition, levels of proinflammatory mediators, including $\mathrm{TNF} \alpha$, IL-1 $\beta$, IL-6, and eicosanoids are elevated in the brains and peripheral blood mononuclear cells (PBMCs) of patients with PD $[6,7]$. Nitrite in the cerebrospinal fluid as well as increased expression of inducible nitric oxide synthase (iNOS) within the SN have been found in PD patients $[9,10]$. All of these findings lend strong support to the association of inflammation and PD. Many of these inflammatory mediators have been demonstrated to have strong neurotoxic effects on DA-neurons $[1,11-14]$, and the central cell implicated in the initiation and execution of inflammatory responses within the CNS is the microglial cell. The pro-inflammatory response of the microglial cell is primarily mediated by the transcription factor NF- $\kappa \mathrm{B}$, which is activated by pro-inflammatory signals and controls 
the gene expression of most of the inflammatory mediators produced by microglial cells. Understanding the role of inflammation in the etiology of $\mathrm{PD}$, developing effective anti-inflammatory therapies directed at NF- $\kappa \mathrm{B}$ activity in microglia, and determining the efficacy of these NF- $\kappa$ B inhibitors in protecting DA-neurons from degeneration are of particular interest as therapeutic approaches aimed at stopping and reversing the debilitating effects of PD.

\section{Microglial Cells, PD, and Mechanisms of Inflammation}

The etiology of PD suggests that chronic production of inflammatory mediators such as $\mathrm{TNF} \alpha$, nitric oxide (NO), and IL- $1 \beta$ mediates a significant amount of neuronal tissue destruction, and the major cell within the CNS that produces these mediators is the microglial cell. Several agents which directly activate microglia have been shown to induce neurotoxicity to DA-producing neurons both in vitro and in vivo. These include LPS [14], f-Met-Leu-Phe (fMLP), $\beta$-amyloid peptides [15], Parkin [16], and aggregated or nitrated- $\alpha$-synuclein [17]. In addition, several direct neurotoxins such as 1-methyl-4-phenyl-1,2,3,6-tetrahydropyridine (MPTP) and 6-hydroxydopamine (6-OHDA) can activate microglia through the release of cellular contents, which leads to an exacerbation of the neurotoxicity [18-21]. There is also a strong correlation between environmental factors which lead to the induction of an inflammatory response within the brain, including traumatic brain injury $[22,23]$ and infection [24], and the ultimate onset of PD. Once activated, microglial cells produce a wide variety of inflammatory mediators which serve to promote an innate immune response, including inflammatory cytokines, chemokines, prostaglandins and leukotrienes, NO, reactive oxygen species (ROS), and glutamate.

Figure 1 proposes our model on the role of inflammation in progressive neurodegeneration like that seen in PD. Pro-inflammatory signals such as infection, trauma, stress, or exposure to environmental factors can directly activate microglial cells. Activated microglial cells, like macrophages, undergo changes that enhance their microbicidal effectiveness and their ability to modulate the inflammatory immune response. These changes include enhanced phagocytic ability, microbial killing, antigen presentation, and inflammatory cytokine production. Activated microglial cells then secrete these inflammatory cytokines, such as TNF- $\alpha$, IL- $1 \beta$, MIP$1 \alpha$, and IL-6 [25], which can both recruit new inflammatory cells as well as lead to direct killing of DA neurons.

Activated microglial cells also release NO, which is produced by inducible nitric oxide synthase (iNOS) [26] Furthermore, microglial cell activation leads to enhanced respiratory burst activity as well. The microglial respiratory burst is characterized by the release of various reactive oxygen species (ROS) including superoxide radicals and hydrogen peroxide. It is now clear that many of the inflammatory mediators produced by activated microglia can actively trigger apoptosis in neuronal cell cultures. These mediators include tumor necrosis factor- $\alpha$ (TNF- $\alpha$ ) [27] nitric oxide $(\mathrm{NO})$, interleukin- $1 \beta$ (IL-1b, cathepsin-B) $[7,28]$, glutamate

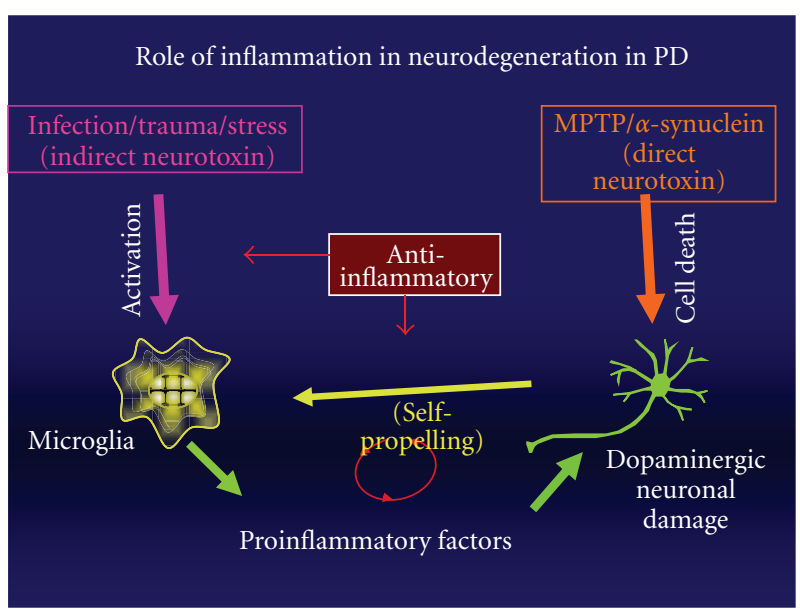

FIGURE 1

[29, 30], IL-8 [31], and ROS [10]. Highly elevated levels of several of these inflammatory mediators (TNF, NO, PGE2, IL- $1 \beta$, IL-6) can be found in the cerebrospinal fluid (CSF) of PD patients, as well as in the brain SNpc tissue of PD patients when analyzed in postmortem autopsies [7, 9, 10, 27, 32, 33]. Interestingly, NF- $\kappa \mathrm{B}$ activation is required for all of these mediators to be produced by microglial cells. In addition, microglial cells can be activated following the death of DAneurons from either inflammatory damage or from the direct effects of DA-neurotoxins such as MPTP or 6-OHDA. It has also been found that intranigral and/or plasma TNF levels remained elevated in MPTP-treated rodents or nonhuman primates one year after administration of the neurotoxin [34]. Once microglial cells have been activated to release neurotoxic inflammatory mediators, further DA neuronal damage occurs which results in the generation of reactive microgliosis, a process by which there is a self-perpetuating cycle resulting in sustained chronic neuroinflammation that drives the progressive neurodegeneration in $\mathrm{PD}$. This process leads to the chronic nature of the disease and to sustained neuronal damage over a prolonged period of time. Therefore, intervention with anti-inflammatory reagents such as NF- $\kappa \mathrm{B}$ inhibitors could function to inhibit the initial activation of microglial cells or to halt the continual reactivation which occurs during reactive microgliosis, which is the hallmark of this chronic inflammatory condition. Ultimately, reactive microgliosis, if left unchecked, will result in destruction of DA-producing neurons.

\section{The Biology of NF- $\kappa$ B}

Many of the inflammatory mediators involved in inflammation and DA neurodegeneration in PD have a common feature: their expression in microglial cells is primarily regulated by NF- $\kappa \mathrm{B}$. The transcription factor NF- $\kappa \mathrm{B}$, first described by David Baltimore's group in 1986 as a transcription factor which is essential for the expression of mouse kappa light chain genes $[35,36]$, has now been found to control gene expression of many of proinflammatory responses. NF- $\kappa \mathrm{B}$ is a "master switch" for inflammatory 
gene expression [37]. Inflammatory cytokines such as TNF and IL- $1 \alpha$ and $\beta$, bacterial products such as lipopolysaccharide (LPS), and products of cellular damage strongly activate inflammatory responses through the activation of NF- $\kappa \mathrm{B}$. NF $\kappa \mathrm{B}$ subsequently plays an essential role in the inflammatory response through regulation of genes encoding inflammatory cytokines (IL-1 $\beta, \mathrm{TNF} \alpha$, IL-12/23), chemokines (IL-8, MIP- $1 \alpha$, MCP-1 [38-40]), nitric oxide production (iNOS), NADPH oxidase subunits $\mathrm{p} 47$ and p67 $[41,42]$, and adhesion molecules (ICAM-1, VCAM, and E-selectin $[43,44])$. Activation of NF- $\kappa \mathrm{B}$ is a key event in many chronic inflammatory diseases such as asthma, cardiovascular disease [45], tissue reperfusion injury [46], experimental autoimmune encephalomyelitis (EAE) [47], rheumatoid arthritis [48], and inflammatory bowel disease (IBD) [49]. Many of the standard agents used to treat human inflammatory conditions, including sulfasalazine, 5aminosalicylates, and corticosteroids, as well as some natural anti-inflammatory compounds such as IL-10, TGF $\beta 1, \beta 2 \mathrm{AR}$ agonists, glutamate, and curcumin, among others, have been postulated to exert some of their anti-inflammatory effects through NF- $\kappa$ B inhibition [41, 50-52]. We and others have found that these compounds are potent inhibitors of microglial activation and are neuroprotective to DA neurons in vitro and/or in vivo. Thus, NF- $\kappa \mathrm{B}$ activity emerges as a key target to control the chronic inflammation in humans, and strategies for its use in PD to inhibit NF- $\kappa \mathrm{B}$ activity in microglial cells more potently may lead to more effective treatments for $\mathrm{PD}$.

The NF- $\kappa$ B family consists of dimeric transcription factors which include five members: c-Rel, RelA (p65), RelB, NF- $\kappa$ B1 (p50/p105), and NF- $\kappa$ B2 (p52/p100). There are two major pathways of activation: the classical or canonical pathway and the alternate or noncanonical pathway. The classical pathway, which is thought to regulate the production of most pro-inflammatory mediators, is mediated through the activation of a dimer of Rel proteins p50 and p65, complexed within the cytosol to the inhibitory complex $\mathrm{I} \kappa \mathrm{B} \alpha[41]$. The activation of the classical NF- $\kappa \mathrm{B}$ pathway is dependent on the phosphorylation, ubiquitination, and subsequent proteasome-dependent degradation of $\mathrm{I} \kappa \mathrm{B} \alpha$. The phosphorylation of $\mathrm{I} \kappa \mathrm{B} \alpha$ on serine residues is mediated by $\mathrm{I} \kappa \mathrm{B}$ kinase (IKK), which is a molecular complex of three proteins consisting of a heterodimer of the two catalytic units IKK $\alpha$ and IKK $\beta$, along with IKK $\gamma$ (the NF- $\kappa$ B essential modulator, NEMO) [53-55]. Mice null for IKK $\beta, \operatorname{IKK} \gamma$, or p65 but not IKK $\alpha$ are embryonic lethal as a result of massive liver apoptosis. Cells derived from these embryos are unresponsive to classical NF- $\kappa \mathrm{B}$ inducers such as TNF $\alpha$ and IL-1 $\beta$ [56-58], demonstrating a signaling link between $\mathrm{p} 65, \operatorname{IKK} \beta$, and IKK $\gamma$ subunits. Activation of the IKK in response to inflammatory mediators like TNF $\alpha$, IL-1 $\beta$, and LPS depends critically on the presence of the IKK $\gamma$ (NEMO) subunit of the IKK complex $[55,59]$ and results in the phosphorylation of the I $\kappa \mathrm{B}$ by the kinase activity of IKK $\beta$ $[53,54]$. An N-terminal region of NEMO associates with a hexapeptide sequence within the C-terminus of both IKK $\alpha$ and IKK $\beta$, named the NEMO-binding domain (NBD), and disruption or mutation of this NEMO-NBD interaction site on either IKK $\beta$ or IKK $\gamma$ results in a loss of responsiveness of cells to pro-inflammatory signaling.

On the other hand, the noncanonical pathway of NF- $\kappa \mathrm{B}$ consists of heterodimers of Rel proteins p100/RelB that also have transcriptional activity but appear to play more of a regulatory role in cellular activation and differentiation rather than in inflammation. In response to a set of factors that include CD40L, B cell-activating factor, and lymphotoxin $-\beta, N F-\kappa B$ is activated through an alternative pathway independent of IKK [60-65]. Instead, activation proceeds through the NF- $\kappa \mathrm{B}$-inducing kinase (NIK) that phosphorylates and activates IKK $\alpha \alpha$ homodimers which, in turn, phosphorylate p100 in complex with RelB. This leads to ubiquitin-dependent processing of p100 to p52 and translocation of p52/RelB to the nucleus $[63,64]$. Cytokineinduced activation of the noncanonical pathway of NF- $\kappa \mathrm{B}$ is accompanied by an increase in the concentration of nuclear IKK $\alpha$ that phosphorylates histone $\mathrm{H} 3[66,67]$. In cells exposed to cytokines, nuclear IKK $\alpha$ regulates gene expression through promoter-associated histone phosphorylation and binding to promoter regions of NF- $\kappa \mathrm{B}$ responsive genes. Mice deficient in IKK $\alpha$ die perinatally, with phenotypical changes of dermal and skeletal development [68-71]. B-cell activating factor, NIK, and p100/p52 knockout mice have similar phenotypes $[72,73]$, suggesting that these molecules are all part of the same linear nonclassical signaling cascade. In addition, the classical and alternative pathways are thought to regulate distinct genes in response to their various activators [65]. Relevant to PD, it has now been found that the canonical pathway is highly activated within the SN of animals undergoing DA neurodegeneration, and aftermortem in the brains of PD patients [74, 75]. On the other hand, the non-canonical NF- $\kappa$ B pathway is found to be activated in regenerating DA-neurons from rats treated with glial-derived neurotrophic factor (GDNF), while the canonical p65/p50 pathway is concomitantly decreased, suggesting that this non-canonical NF- $\kappa \mathrm{B}$ pathway is important in neuron regeneration of DA neurons within the SN [76].

\section{Therapeutic Usage of Specific NF- $\kappa$ B Inhibitors in Chronic Inflammation}

Due to the central role of the IKK $\gamma$ and Ikk $\beta$ molecules within the IKK complex in activating inflammation, the identification of selective IKK $\beta$ and IKK $\gamma$ inhibitors that do not target IKK $\alpha$ or the P100/p52 pathway as therapeutic agents in treating chronic inflammation is of considerable interest. Two specific inhibitors of NF- $\kappa \mathrm{B}$ have emerged that appear to be highly therapeutically active in the treatment of several chronic inflammatory diseases, and which provide possible therapeutic approaches to the treatment of PD. The first is a peptide directed against the $\mathrm{N}$-terminal region of NEMO that associates with a hexapeptide sequence within the C-terminus of both IKK $\alpha$ and IKK $\beta$, named the NEMObinding domain (NBD). This cell permeable peptide spans the NBD and disrupts the association of NEMO with IKKs in vitro and blocks TNF $\alpha$-induced NF- $\kappa \mathrm{B}$ activation in vivo [77, 78]. Notably, the NBD peptide does not affect basal activity of the IKK but only suppresses the induction of activity 
in response to inflammatory cytokines [77]. Continuous administration of the NBD peptide effectively ameliorates inflammatory responses in animal models without overt signs of toxicity [78]. Additionally, in mouse models of chronic inflammation, including collagen-induced arthritis (CIA) [79, 80], experimental allergic encephalomyelitis (EAE), Duchenne's muscular dystrophy (Peterson et al., manuscript in submission), and inflammatory bowel disease (IBD) [81], in vivo treatment with NBD peptides blocked disease activity, inflammatory cytokine expression, and homing of cells to inflammatory sites. Furthermore, mice treated systemically with an NBD peptide for five days after induction of CIA maintained clinical and histological improvement for nearly three weeks following termination of peptide administration [79]. It is important to note that in the therapeutic use of NBD peptide, there was no evidence of undesired off-target effects as the treatment with NBD peptide was shown to be specific for inhibiting NF$\kappa \mathrm{B}$ signaling, exhibiting no inhibitory effects towards JNK or p38 MAPK pathways [78]. The safety profile for NBD is favorable as well. In vivo, systemic delivery of NBD is not associated with any described toxicity in mice or rats, and inhibition of NF- $\kappa$ B has been demonstrated to ameliorate an ever-growing list of inflammatory disease conditions [77$79,82,83]$. Therefore, the therapeutic effect of the short-lived NBD peptide may far outlast its pharmacokinetic properties. Thus, selective IKK inhibition by NBD peptides may (i) be an effective therapeutic intervention in chronic inflammatory diseases; (ii) lead to durable alterations in immune responses that correlate with durable clinical efficacy; (iii) minimize potential toxicity concerns associated as basal NF- $\kappa$ B activity remains intact as does the alternative pathway of $\mathrm{NF}-\kappa \mathrm{B}$ activation necessary for B-cell development and lymphoid organogenesis.

A second approach to the inhibition of inflammation has been to utilize small molecule inhibitors that specifically block the kinase enzymatic activity of IKK $\beta$. One such specific inhibitor, called Compound $A$, is a small molecule inhibitor of the kinase activity of IKK $\beta$ but not IKK $\alpha$. Compound A, also known as BAY-65-1942 (7-[2-(cyclopropylmethoxy)-6-hydroxyphenyl]-5-[(3S)-3-piperidinyl]1,4-dihydro-2Hpyrido [2,3-d] [1,3]-oxazin-2-one hydrochloride), has been shown to specifically and effectively block the catalytic activity of IKK $\beta$, inhibiting its ability to phosphorylate $\mathrm{I} \kappa \mathrm{B}$ and activate the cytosolic p50/p65 NF- $\kappa$ B heterodimers $[84,85]$. Compound $\mathrm{A}$ has been used extensively in vivo, and it has now been found to prevent pulmonary inflammation [86], to attenuate myocardial injury and dysfunction after ischemia-reperfusion injury [84], and to prevent graft versus host disease in murine models of GVHD (Serody et al., personal communication). Other IKK $\beta$ inhibitors, including PS-1145 [87] and TPCA-1 [88], have been shown to effectively prevent graft versus host disease in a murine bone marrow transplant model [89], to enhance sensitivity of multiple myeloma cells to chemotherapy by inhibiting the protective effects of NF- $\kappa \mathrm{B}$ [90], to inhibit melanoma growth in vivo [91-93], and to inhibit the growth of colon cancer [94]. These inhibitors work primarily through the inhibition of IKK $\beta$, and their specific suppression of the canonical NF- $\kappa \mathrm{B}$ signaling pathway and consequent decrease in serum levels of TNF $\alpha$ and IL- 6 are the main features which mediate their inhibitory activity.

\section{NF- $\kappa$ B and DA Neuroinflammation}

We have found that a number of anti-inflammatory compounds have been demonstrated to have efficacy in protecting DA-neurons from degeneration mediated by inflammatory damage. These compounds include IL-10 $[95,96]$, TGF $\beta 1[97,98]$, morphinan derivatives [99], and DPI, an inhibitor of oxidative stress responses [100]. It is well known that while these compounds have numerous regulatory effects on multiple biological targets, one of their primary anti-inflammatory features is the inhibition of NF$\kappa \mathrm{B}$ within macrophages and microglial cells [101]. Recently, it has been demonstrated that several other compounds, including pioglitazone (a PPAR $\gamma$ agonist), curcumin [102], and salmeterol (a $\beta 2 A R$ agonist, our reference [103]), function to inhibit DA neurodegeneration by inhibiting NF- $\kappa \mathrm{B}$. However, the most compelling data suggesting a central role for $\mathrm{NF}-\kappa \mathrm{B}$ as a regulatory target for PD therapy comes from the use of specific NF- $\kappa \mathrm{B}$ inhibitors, which have recently been used in murine models of PD to determine if therapeutic administration of these inhibitors could halt the progression of neurodegeneration induced by the neurotoxin MPTP [104] or by activation of CNS inflammation by the intracranial injection of LPS [105]. Compounds that block the activation of NF- $\kappa \mathrm{B}$ are capable of inhibiting the two major inflammatory pathways in microglia-activation of oxidative stress and production of inflammatory mediators, including cytokines TNF $\alpha$, IL- $1 \beta$, and IL-6, as well as chemokines associated with inflammation [101]. Ghosh et al. have used the NBD peptide and a mutant peptide control to study the efficacy of NF- $\kappa \mathrm{B}$ inhibition in stopping or reversing the neurodegenerative effects of MPTP administration in a murine model of PD [104]. Evidence shows that there is a marked increase in NF- $\kappa \mathrm{B}$ activation within the midbrain of animals undergoing neurodegeneration as a result of MPTP administration, as well as in the SNpc of PD patients $[75,104]$, and this activation occurs in the TH+ DA-neurons and in astrocytes and microglia. Administration of NBD peptide but not the mutant control peptide was shown to inhibit MPP+ induced NF- $\kappa \mathrm{B}$ activation in vitro in microglia, astrocytes, as well as in BV-2 microglial cells, as determined by DNA binding and transcriptional activity. More importantly, administration of NBD peptide but not the mutant peptide prior to the injection of MPTP in vivo significantly inhibits the activation of $\mathrm{NF}-\kappa \mathrm{B}$ within the midbrain region. This inhibition of NF- $\kappa \mathrm{B}$ activation is accompanied by a concomitant reduction in inflammatory mediator mRNA expression within the $\mathrm{SNpc}$, as well as the expression of activation markers CD11b and GFAP by microglia and astrocytes, respectively. Mice receiving NBD peptide but not mutant peptide prior to MPTP injection also showed highly significant protection of the nigrostriatum from MPTPinduced neurodegeneration of the $\mathrm{TH}+$ neurons and the loss of dopamine production, as well as improvement in their 
locomotor function compared with MPTP-injected mice given mutant peptide. More importantly, administration of NBD peptide 2 days subsequent to the injection of MPTP shows substantial protection of $\mathrm{TH}+$ neurons, suggesting that NBD peptide can be used therapeutically to slow down or halt the progression of DA neurodegeneration in MPTPtreated animals [104]. Infrared analysis of the brains of NBDtreated animals determined that peptide could be found within the brain tissue in significant quantities, suggesting that the NBD peptide could cross the blood-brain barrier (BBB) and reach the site of inflammation. It remains to be determined the mechanism of NF- $\kappa \mathrm{B}$ inhibition within the $\mathrm{SNpc}$, but these data suggest that NF- $\kappa \mathrm{B}$ is a viable target for therapy for PD patients.

Subsequently, we have used the IKK $\beta$ inhibitor compound A in LPS-induced neurodegeneration to determine if, similar to NBD peptide, inhibition of the canonical NF- $\kappa$ B pathway could halt inflammation-induced DA neurodegeneration. In this model, LPS is injected directly into one side of the midbrain of rats, leading to inflammation-induced degeneration of DA-neurons [105]. It was found that IKK $\beta$ inhibitor compound A was capable of strongly inhibiting the activation of NF- $\kappa \mathrm{B}$ in vitro and in vivo, as well as the mRNA expression and subsequent release of pro-inflammatory mediators. Compound A also significantly inhibited LPSand MPTP-induced DA neurotoxicity in vitro, and this neuroprotective activity required the presence of microglial cells. Most importantly, administration of compound A to animals injected intranigrally with LPS attenuated LPS injection-induced DA neuronal loss and microglia activation within the SNpc [105]. Taken together, these data provide strong evidence that NF- $\kappa \mathrm{B}$ offers an excellent therapeutic target to inhibit DA neurodegeneration, and that significant additional work needs to be performed to determine the optimal approach and agent best suited for the treatment of PD.

\section{Conclusion}

Strong evidence now exists that inflammation plays a key role in the neurodegeneration seen in $\mathrm{PD}$, and that effective anti-inflammatory therapy can be very useful in slowing down or preventing the chronic destruction of DA-neurons which is the hallmark of the disease. While a number of anti-inflammatories have already been developed and utilized, many of these compounds have multiple off-target effects or do not show strong efficacy in the treatment of PD. In addition, it is not yet clear the best target for anti-inflammatory therapy in PD and other chronic inflammatory conditions. NF- $\kappa \mathrm{B}$, the "master switch" of inflammation, offers an ideal target for therapy because of its key role in the production of the inflammatory mediators known to exhibit DA neurotoxicity in vitro and in vivo. Targeting the activation pathway of NF- $\kappa$ B by inhibiting the IKK complex has now been shown to be a highly effective therapy for the treatment of neurodegeneration in murine models of PD and offers us a new avenue of investigation towards the development of more effective therapies aimed at stopping and reversing DA-neuron loss in this disease.

\section{References}

[1] M. L. Block and J. S. Hong, "Microglia and inflammationmediated neurodegeneration: multiple triggers with a common mechanism," Progress in Neurobiology, vol. 76, no. 2, pp. 77-98, 2005.

[2] A. L. Bartels and K. L. Leenders, "Neuroinflammation in the pathophysiology of Parkinson's disease: evidence from animal models to human in vivo studies with [C]-PK11195 PET," Movement Disorders, vol. 22, no. 13, pp. 1852-1856, 2007.

[3] W. Dauer and S. Przedborski, "Parkinson's disease: mechanisms and models," Neuron, vol. 39, no. 6, pp. 889-909, 2003.

[4] H. M. Gao and J. S. Hong, "Why neurodegenerative diseases are progressive: uncontrolled inflammation drives disease progression," Trends in Immunology, vol. 29, no. 8, pp. 357365, 2008.

[5] P. L. McGeer, S. Itagaki, B. E. Boyes, and E. G. McGeer, "Reactive microglia are positive for HLA-DR in the substantia nigra of Parkinson's and Alzheimer's disease brains," Neurology, vol. 38, no. 8, pp. 1285-1291, 1988.

[6] T. Nagatsu, M. Mogi, H. Ichinose, and A. Togari, "Changes in cytokines and neurotrophins in Parkinson's disease," Journal of Neural Transmission, no. 60, supplement, pp. 277-290, 2000.

[7] M. Mogi, M. Harada, T. Kondob et al., "Interleukin-1 $\beta$, interleukin-6, epidermal growth factor and transforming growth factor- $\alpha$ are elevated in the brain from parkinsonian patients," Neuroscience Letters, vol. 180, no. 2, pp. 147-150, 1994.

[8] Y. Ouchi, S. Yagi, M. Yokokura, and M. Sakamoto, "Neuroinflammation in the living brain of Parkinson's disease," Parkinsonism and Related Disorders, vol. 15, no. 3, pp. S200S204, 2009.

[9] G. A. Qureshi, S. Baig, I. Bednar, P. Sodersten, G. Forsberg, and A. Siden, "Increased cerebrospinal fluid concentration of nitrite ire Parkinson's disease," NeuroReport, vol. 6, no. 12, pp. 1642-1644, 1995.

[10] S. Hunot, F. Boissière, B. Faucheux et al., "Nitric oxide synthase and neuronal vulnerability in Parkinson's disease," Neuroscience, vol. 72, no. 2, pp. 355-363, 1996.

[11] L. Qian and P. M. Flood, "Microglial cells and Parkinson's disease," Immunologic Research, vol. 41, no. 3, pp. 155-164, 2008.

[12] M. K. McCoy and M. G. Tansey, "TNF signaling inhibition in the CNS: implications for normal brain function and neurodegenerative disease," Journal of Neuroinflammation, vol. 5, article 45, 2008.

[13] P. Jenner, "Oxidative mechanisms in nigral cell death in Parkinson's disease," Movement Disorders, vol. 13, supplement 1, pp. 24-34, 1998.

[14] J. Zielasek and H. P. Hartung, "Molecular mechanisms of microglial activation," Advances in Neuroimmunology, vol. 6, no. 2, pp. 191-222, 1996.

[15] J. Rogers and L. F. Lue, "Microglial chemotaxis, activation, and phagocytosis of amyloid $\beta$-peptide as linked phenomena in Alzheimer's disease," Neurochemistry International, vol. 39, no. 5-6, pp. 333-340, 2001.

[16] T. C. Frank-Cannon, T. Tran, K. A. Ruhn et al., "Parkin deficiency increases vulnerability to inflammation-related nigral degeneration," Journal of Neuroscience, vol. 28, no. 43, pp. 10825-10834, 2008. 
[17] A. D. Reynolds, J. G. Glanzer, I. Kadiu et al., "Nitrated alphasynuclein-activated microglial profiling for Parkinson's disease," Journal of Neurochemistry, vol. 104, no. 6, pp. 15041525, 2008.

[18] E. C. Hirsch, S. Hunot, and A. Hartmann, "Neuroinflammatory processes in Parkinson's disease," Parkinsonism and Related Disorders, vol. 11, no. 1, pp. S9-S15, 2005.

[19] H. Wilms, L. Zecca, P. Rosenstiel, J. Sievers, G. Deuschl, and R. Lucius, "Inflammation in Parkinson's diseases and other neurodegenerative diseases: cause and therapeutic implications," Current Pharmaceutical Design, vol. 13, no. 18, pp. 1925-1928, 2007.

[20] P. L. McGeer, C. Schwab, A. Parent, and D. Doudet, "Presence of reactive microglia in monkey Substantia nigra years after 1-methyl-4-phenyl-1,2,3,6-tetrahydropyridine administration," Annals of Neurology, vol. 54, no. 5, pp. 599-604, 2003.

[21] F. Cicchetti, A. L. Brownell, K. Williams, Y. I. Chen, E. Livni, and O. Isacson, "Neuroinflammation of the nigrostriatal pathway during progressive 6-OHDA dopamine degeneration in rats monitored by immunohistochemistry and PET imaging," European Journal of Neuroscience, vol. 15, no. 6, pp. 991-998, 2002.

[22] W. Meissner, C. Prunier, D. Guilloteau, S. Chalon, C. E. Gross, and E. Bezard, "Time-course of nigrostriatal degeneration in a progressive MPTP-lesioned macaque model of Parkinson's disease," Molecular Neurobiology, vol. 28, no. 3, pp. 209-218, 2003.

[23] A. D. Ebert, J. H. Hoo, and M. C. Bohn, "Progressive degeneration of dopamine neurons in 6-hydroxydopamine rat model of Parkinson's disease does not involve activation of caspase-9 and caspase-3," Journal of Neuroscience Research, vol. 86, no. 2, pp. 317-325, 2008.

[24] A. R. Simard and S. Rivest, "Do pathogen exposure and innate immunity cause brain diseases?" Neurological Research, vol. 27, no. 7, pp. 717-725, 2005.

[25] R. M. Ransohoff and V. H. Perry, "Microglial physiology: unique stimuli, specialized responses," Annual Review of Immunology, vol. 27, pp. 119-145, 2009.

[26] G. C. Brown and J. J. Neher, "Inflammatory neurodegeneration and mechanisms of microglial killing of neurons," Molecular Neurobiology, vol. 41, no. 2-3, pp. 242-247, 2010.

[27] M. Mogi, M. Harada, P. Riederer, H. Narabayashi, K. Fujita, and T. Nagatsu, "Tumor necrosis factor- $\alpha$ (TNF- $\alpha$ ) increases both in the brain and in the cerebrospinal fluid from parkinsonian patients," Neuroscience Letters, vol. 165, no. 1-2, pp. 208-210, 1994.

[28] H. Arai, T. Furuya, T. Yasuda, M. Miura, Y. Mizuno, and H. Mochizuki, "Neurotoxic effects of lipopolysaccharide on nigral dopaminergic neurons are mediated by microglial activation, interleukin- $1 \beta$, and expression of caspase- 11 in mice," Journal of Biological Chemistry, vol. 279, no. 49, pp. 51647-51653, 2004.

[29] W. M. Caudle and J. Zhang, "Glutamate, excitotoxicity, and programmed cell death in parkinson disease," Experimental Neurology, vol. 220, no. 2, pp. 230-233, 2009.

[30] D. W. Choi, "Glutamate neurotoxicity and diseases of the nervous system," Neuron, vol. 1, no. 8, pp. 623-634, 1988.

[31] L. Thirumangalakudi, L. Yin, H. V. Rao, and P. Grammas, "IL-8 induces expression of matrix metalloproteinases, cell cycle and pro-apoptotic proteins, and cell death in cultured neurons," Journal of Alzheimer's Disease, vol. 11, no. 3, pp. 305-311, 2007.
[32] D. A. Loeffler, A. J. DeMaggio, P. L. Juneau, M. K. Havaich, and P. A. LeWitt, "Effects of enhanced striatal dopamine turnover in vivo on glutathione oxidation," Clinical Neuropharmacology, vol. 17, no. 4, pp. 370-379, 1994.

[33] K. Imamura, N. Hishikawa, M. Sawada, T. Nagatsu, M. Yoshida, and Y. Hashizume, "Distribution of major histocompatibility complex class II-positive microglia and cytokine profile of Parkinson's disease brains," Acta Neuropathologica, vol. 106, no. 6, pp. 518-526, 2003.

[34] C. Barcia, V. De Pablos, V. Bautista-Hernández et al., "Increased plasma levels of TNF- $\alpha$ but not of IL1- $\beta$ in MPTPtreated monkeys one year after the MPTP administration," Parkinsonism and Related Disorders, vol. 11, no. 7, pp. 435439, 2005.

[35] R. Sen and D. Baltimore, "Multiple nuclear factors interact with the immunoglobulin enhancer sequences," Cell, vol. 46, no. 5, pp. 705-716, 1986.

[36] R. Sen and D. Baltimore, "Inducibility of K immunoglobulin enhancer-binding protein NF- $\kappa$ B by a posttranslational mechanism," Cell, vol. 47, no. 6, pp. 921-928, 1986.

[37] G. Tsoulfas and D. A. Geller, "NF- $\kappa$ B in transplantation: friend or foe?" Transplant Infectious Disease, vol. 3, no. 4, pp. 212-219, 2001.

[38] K. A. Roebuck, "Regulation of interleukin-8 gene expression," Journal of Interferon and Cytokine Research, vol. 19, no. 5, pp. 429-438, 1999.

[39] Y. Xia, M. E. Pauza, L. Feng, and D. Lo, "RelB regulation of chemokine expression modulates local inflammation," American Journal of Pathology, vol. 151, no. 2, pp. 375-387, 1997.

[40] K. A. Roebuck, L. R. Carpenter, V. Lakshminarayanan, S. M. Page, J. N. Moy, and L. L. Thomas, "Stimulus-specific regulation of chemokine expression involves differential activation of the redox-responsive transcription factors AP1 and NF- $\kappa$ B," Journal of Leukocyte Biology, vol. 65, no. 3, pp. 291-298, 1999.

[41] T. Lawrence, "The nuclear factor NF-kappaB pathway in inflammation," Cold Spring Harbor Perspectives in Biology, vol. 1, no. 6, article a001651, 2009.

[42] K. A. Gauss, L. K. Nelson-Overton, D. W. Siemsen, Y. Gao, F. R. DeLeo, and M. T. Quinn, "Role of NF- $\kappa$ B in transcriptional regulation of the phagocyte NADPH oxidase by tumor necrosis factor- $\alpha$, Journal of Leukocyte Biology, vol. 82, no. 3, pp. 729-741, 2007.

[43] C. C. Chen and A. M. Manning, "Transcriptional regulation of endothelial cell adhesion molecules: a dominant role for NF- $\kappa \mathrm{B}$," Agents and Actions Supplements, vol. 47, pp. 135141, 1995.

[44] P. P. Tak and G. S. Firestein, "NF- $\kappa$ B: a key role in inflammatory diseases," Journal of Clinical Investigation, vol. 107, no. 1, pp. 7-11, 2001.

[45] K. Van der Heiden, S. Cuhlmann, A. Luong, M. Zakkar, and P. C. Evans, "Role of nuclear factor kappaB in cardiovascular health and disease," Clinical Science, vol. 118, no. 10, pp. 593605, 2010.

[46] C. A. Latanich and L. H. Toledo-Pereyra, "Searching for NF$\kappa \mathrm{B}$-based treatments of ischemia reperfusion injury," Journal of Investigative Surgery, vol. 22, no. 4, pp. 301-315, 2009.

[47] K. Vandenbroeck, I. Alloza, M. Gadina, and P. Matthys, "Inhibiting cytokines of the interleukin-12 family: recent advances and novel challenges," Journal of Pharmacy and Pharmacology, vol. 56, no. 2, pp. 145-160, 2004. 
[48] L. A. Criswell, "Gene discovery in rheumatoid arthritis highlights the CD40/NF- $\kappa \mathrm{B}$ signaling pathway in disease pathogenesis," Immunological Reviews, vol. 233, no. 1, pp. 5561, 2010.

[49] I. Atreya, R. Atreya, and M. F. Neurath, "NF- $\kappa$ B in inflammatory bowel disease," Journal of Internal Medicine, vol. 263, no. 6, pp. 591-596, 2008.

[50] T. Lawrence and C. Fong, "The resolution of inflammation: anti-inflammatory roles for NF- $\kappa$ B," International Journal of Biochemistry and Cell Biology, vol. 42, no. 4, pp. 519-523, 2010.

[51] S. G. Pereira and F. Oakley, "Nuclear factor- $\kappa$ B1: regulation and function," International Journal of Biochemistry and Cell Biology, vol. 40, no. 8, pp. 1425-1430, 2008.

[52] M. S. Wang, S. Boddapati, S. Emadi, and M. R. Sierks, "Curcumin reduces $\alpha$-synuclein induced cytotoxicity in Parkinson's disease cell model," BMC Neuroscience, vol. 11, article 57, 2010.

[53] T. Huxford and G. Ghosh, "A structural guide to proteins of the NF-kappaB signaling module," Cold Spring Harbor Perspectives in Biology, vol. 1, no. 3, article a000075, 2009.

[54] A. Oeckinghaus and S. Ghosh, "The NF-kappaB family of transcription factors and its regulation," Cold Spring Harbor Perspectives in Biology, vol. 1, no. 4, article a000034, 2009.

[55] M. J. May, R. B. Marienfeld, and S. Ghosh, "Characterization of the I $\kappa$ B-kinase NEMO binding domain," Journal of Biological Chemistry, vol. 277, no. 48, pp. 45992-46000, 2002.

[56] N. Sizemore et al., "Distinct roles of the Ikappa B kinase alpha and beta subunits in liberating nuclear factor kappa $B$ (NF-kappa B) from Ikappa B and in phosphorylating the p65 subunit of NF-kappa B," The Journal of Biological Chemistry, vol. 277, no. 6, pp. 3863-3869, 2002.

[57] N. Sizemore, A. Agarwal, K. Das et al., "Inhibitor of $\kappa$ B kinase is required to activate a subset of interferon $\gamma$-stimulated genes," Proceedings of the National Academy of Sciences of the United States of America, vol. 101, no. 21, pp. 7994-7998, 2004.

[58] J. Y. Reuther-Madrid, D. Kashatus, S. Chen et al., "The p65/RelA subunit of NF- $\kappa$ B suppresses the sustained, antiapoptotic activity of Jun kinase induced by tumor necrosis factor," Molecular and Cellular Biology, vol. 22, no. 23, pp. 8175-8183, 2002.

[59] D. Rudolph, W. C. Yeh, A. Wakeham et al., "Severe liver degeneration and lack of NF- $\kappa$ B activation in NEMO/IKK $\gamma$ deficient mice," Genes and Development, vol. 14, no. 7, pp. 854-862, 2000.

[60] Z. P. Xia and Z. J. Chen, "TRAF2: a double-edged sword?" Science's STKE, vol. 2005, no. 272, p. e7, 2005.

[61] M. Neumann and M. Naumann, "Beyond I $\kappa$ Bs: alternative regulation of NF- $\kappa$ B activity," FASEB Journal, vol. 21, no. 11, pp. 2642-2654, 2007.

[62] K. D. Brown, E. Claudio, and U. Siebenlist, "The roles of the classical and alternative nuclear factor- $\kappa \mathrm{B}$ pathways: potential implications for autoimmunity and rheumatoid arthritis," Arthritis Research and Therapy, vol. 10, no. 4, article 212, 2008.

[63] A. Israel, "The IKK complex, a central regulator of NFkappaB activation," Cold Spring Harbor Perspectives in Biology, vol. 2, no. 3, article a000158, 2010.

[64] N. Bakkar and D. C. Guttridge, "NF- $\kappa$ B signaling: a tale of two pathways in skeletal myogenesis," Physiological Reviews, vol. 90, no. 2, pp. 495-511, 2010.

[65] J. L. Pomerantz and D. Baltimore, "Two pathways to NF- $\kappa$ B," Molecular Cell, vol. 10, no. 4, pp. 693-695, 2002.
[66] Y. Yamamoto, U. N. Verma, S. Prajapati, K. Youn-Tae, and R. B. Gaynor, "Histone H3 phosphorylation by ikk- $\alpha$ is critical for cytokine-induced gene expression," Nature, vol. 423, no. 6940, pp. 655-659, 2003.

[67] V. Anest, J. L. Hanson, P. C. Cogswell, K. A. Steinbrecher, B. D. Strahl, and A. S. Baldwin, "A nucleosomal function for $I \kappa B$ kinase- $\alpha$ in NF- $\kappa$ B-dependent gene expression," Nature, vol. 423, no. 6940, pp. 659-663, 2003.

[68] A. K. Sil, S. Maeda, Y. Sono, D. B. Roop, and M. Karin, "I $\kappa \mathrm{B}$ kinase- $\alpha$ acts in the epidermis to control skeletal and craniofacial morphogenesis," Nature, vol. 428, no. 6983, pp. 660-664, 2004.

[69] R. Gareus, M. Huth, B. Breiden et al., "Normal epidermal differentiation but impaired skin-barrier formation upon keratinocyte-restricted IKK1 ablation," Nature Cell Biology, vol. 9, no. 4, pp. 461-469, 2007.

[70] J. M. Dahlman, N. Bakkar, W. He, and D. C. Guttridge, "NF$\kappa \mathrm{B}$ functions in stromal fibroblasts to regulate early postnatal muscle development," Journal of Biological Chemistry, vol. 285, no. 8, pp. 5479-5487, 2010.

[71] N. Bakkar, J. Wang, K. J. Ladner et al., "IKK/NF- $\kappa$ B regulates skeletal myogenesis via a signaling switch to inhibit differentiation and promote mitochondrial biogenesis," Journal of Cell Biology, vol. 180, no. 4, pp. 787-802, 2008.

[72] Y. Xue, X. Wang, Z. Li, N. Gotoh, D. Chapman, and E. Y. Skolnik, "Mesodermal patterning defect in mice lacking the Ste20 NCK interacting kinase (NIK)," Development, vol. 128, no. 9, pp. 1559-1572, 2001.

[73] N. S. Soysa, N. Alles, D. Weih et al., "The pivotal role of the alternative NF- $\kappa \mathrm{B}$ pathway in maintenance of basal bone homeostasis and osteoclastogenesis," Journal of Bone and Mineral Research, vol. 25, no. 4, pp. 809-818, 2010.

[74] M. Mogi, T. Kondo, Y. Mizuno, and T. Nagatsu, "p53 protein, interferon $-\gamma$, and NF- $\kappa$ B levels are elevated in the parkinsonian brain," Neuroscience Letters, vol. 414, no. 1, pp. 94-97, 2007.

[75] S. Hunot, B. Brugg, D. Ricard et al., "Nuclear translocation of NF- $\kappa \mathrm{b}$ is increased in dopaminergic neurons of patients with Parkinson disease," Proceedings of the National Academy of Sciences of the United States of America, vol. 94, no. 14, pp. 7531-7536, 1997.

[76] J. P. Cao, H. J. Wang, J. K. Yu, H. M. Liu, and D. S. Gao, "The involvement of NF- $\kappa$ B p65/p52 in the effects of GDNF on DA neurons in early PD rats," Brain Research Bulletin, vol. 76, no. 5, pp. 505-511, 2008.

[77] M. J. May, F. D’Acquisto, L. A. Madge, J. Glockner, J. S. Pober, and S. Ghosh, "Selective inhibition of NF- $\kappa$ B activation by a peptide that blocks the interaction of NEMO with the I $\kappa$ B kinase complex," Science, vol. 289, no. 5484, pp. 1550-1554, 2000.

[78] P. Di Meglio, A. Ianaro, and S. Ghosh, "Amelioration of acute inflammation by systemic administration of a cellpermeable peptide inhibitor of NF- $\kappa \mathrm{B}$ activation," Arthritis and Rheumatism, vol. 52, no. 3, pp. 951-958, 2005.

[79] E. Jimi, K. Aoki, H. Saito et al., "Selective inhibition of NF- $\kappa$ B blocks osteoclastogenesis and prevents inflammatory bone destruction in vivo," Nature Medicine, vol. 10, no. 6, pp. 617624, 2004.

[80] S. Dai, T. Hirayama, S. Abbas, and Y. Abu-Amer, "The I $\kappa$ B kinase (IKK) inhibitor, NEMO-binding domain peptide, blocks osteoclastogenesis and bone erosion in inflammatory arthritis," Journal of Biological Chemistry, vol. 279, no. 36, pp. 37219-37222, 2004. 
[81] S. H. Davé, J. S. Tilstra, K. Matsuoka et al., "Amelioration of chronic murine colitis by peptide-mediated transduction of the I $\kappa \mathrm{B}$ kinase inhibitor NEMO binding domain peptide," Journal of Immunology, vol. 179, no. 11, pp. 7852-7859, 2007.

[82] A. Desai, N. Singh, and R. Raghubir, "Neuroprotective potential of the NF-[kappa]B inhibitor peptide IKK-NBD in cerebral ischemia-reperfusion injury," Neurochemistry International, vol. 57, no. 8, pp. 876-883, 2010.

[83] G. Grassia et al., "The IkappaB kinase inhibitor nuclear factor-kappaB essential modulator-binding domain peptide for inhibition of balloon injury-induced neointimal formation," Arteriosclerosis, Thrombosis, and Vascular Biology, vol. 30, no. 12, pp. 2458-2466, 2010.

[84] N. C. Moss, W. E. Stansfield, M. S. Willis, R. H. Tang, and C. H. Selzman, "IKK $\beta$ inhibition attenuates myocardial injury and dysfunction following acute ischemia-reperfusion injury," American Journal of Physiology, vol. 293, no. 4, pp. H2248-H2253, 2007.

[85] F. Zhang, L. Qian, P. M. Flood, J. S. Shi, J. S. Hong, and H. M. Gao, "Inhibition of I $\kappa$ B kinase- $\beta$ protects dopamine neurons against lipopolysaccharide-induced neurotoxicity," Journal of Pharmacology and Experimental Therapeutics, vol. 333, no. 3, pp. 822-833, 2010.

[86] K. Ziegelbauer, F. Gantner, N. W. Lukacs et al., "A selective novel low-molecular-weight inhibitor of I $\kappa$ B kinase- $\beta$ (IKK$\beta$ ) prevents pulmonary inflammation and shows broad antiinflammatory activity," British Journal of Pharmacology, vol. 145, no. 2, pp. 178-192, 2005.

[87] T. Hideshima, D. Chauhan, P. Richardson et al., "NF- $\kappa$ B as a therapeutic target in multiple myeloma," Journal of Biological Chemistry, vol. 277, no. 19, pp. 16639-16647, 2002.

[88] P. L. Podolin, J. F. Callahan, B. J. Bolognese et al., "Attenuation of murine collagen-induced arthritis by a novel, potent, selective small molecule inhibitor of $\mathrm{I} \kappa \mathrm{B}$ kinase 2, TPCA-1 (2-[(aminocarbonyl)amino]-5-(4-fluorophenyl)-3thiophenecarboxamide), occurs via reduction of proinflammatory cytokines and antigen-induced T cell proliferation," Journal of Pharmacology and Experimental Therapeutics, vol. 312, no. 1, pp. 373-381, 2005.

[89] M. J. O’Shaughnessy, C. Vogtenhuber, K. Sun et al., "Ex vivo inhibition of NF- $\kappa$ B signaling in alloreactive T-cells prevents graft-versus-host disease," American Journal of Transplantation, vol. 9, no. 3, pp. 452-462, 2009.

[90] T. Hideshima, T. Hayashi, D. Chauhan, M. Akiyama, P. Richardson, and K. Anderson, "Biologic sequelae of c-Jun NH-terminal kinase (JNK) activation in multiple myeloma cell lines," Oncogene, vol. 22, no. 54, pp. 8797-8801, 2003.

[91] J. Yang et al., "Conditional ablation of Ikkb inhibits melanoma tumor development in mice," The Journal of Clinical Investigation, vol. 120, no. 7, pp. 2563-2574, 2010.

[92] M. Schön, B. G. Wienrich, S. Kneitz et al., "KINK-1, a novel small-molecule inhibitor of IKK $\beta$, and the susceptibility of melanoma cells to antitumoral treatment," Journal of the National Cancer Institute, vol. 100, no. 12, pp. 862-875, 2008.

[93] K. Amschler, M. P. Schön, N. Pletz, K. Wallbrecht, L. Erpenbeck, and M. Schön, "NF- $\kappa$ B inhibition through proteasome inhibition or ikk $\beta$ blockade increases the susceptibility of melanoma cells to cytostatic treatment through distinct pathways," Journal of Investigative Dermatology, vol. 130, no. 4, pp. 1073-1086, 2010.

[94] A. Yemelyanov, A. Gasparian, P. Lindholm et al., "Effects of IKK inhibitor PS1145 on NF- $\kappa$ B function, proliferation, apoptosis and invasion activity in prostate carcinoma cells," Oncogene, vol. 25, no. 3, pp. 387-398, 2006.
[95] K. W. Moore, R. De Waal Malefyt, R. L. Coffman, and A. O'Garra, "Interleukin-10 and the interleukin-10 receptor," Annual Review of Immunology, vol. 19, pp. 683-765, 2001.

[96] K. Strle, J. H. Zhou, W. H. Shen et al., "Interleukin-10 in the brain," Critical Reviews in Immunology, vol. 21, no. 5, pp. 427-449, 2001.

[97] Y. Zhu, G. Y. Yang, B. Ahlemeyer et al., "Transforming growth factor- $\beta 1$ increases bad phosphorylation and protects neurons against damage," Journal of Neuroscience, vol. 22, no. 10, pp. 3898-3909, 2002.

[98] M. Szczepanik, M. Tutaj, K. Bryniarski, and B. N. Dittel, "Epicutaneously induced TGF- $\beta$-dependent tolerance inhibits experimental autoimmune encephalomyelitis," Journal of Neuroimmunology, vol. 164, no. 1-2, pp. 105-114, 2005.

[99] W. Zhang, J. S. Hong, H. C. Kim, W. Zhang, and M. L. Block, "Morphinan neuroprotection: new insight into the therapy of neurodegeneration," Critical Reviews in Neurobiology, vol. 16, no. 4, pp. 271-302, 2004.

[100] L. Qian et al., "NADPH oxidase inhibitor DPI is neuroprotective at femtomolar concentrations through inhibition of microglia over-activation," Parkinsonism and Related Disorders, vol. 13, supplement 3, pp. S316-S320, 2007.

[101] L. Qian, P. M. Flood, and J. S. Hong, "Neuroinflammation is a key player in Parkinson's disease and a prime target for therapy," Journal of Neural Transmission, vol. 117, no. 8, pp. 971-979, 2010.

[102] S. Yang, D. Zhang, Z. Yang et al., "Curcumin protects dopaminergic neuron against LPS induced neurotoxicity in primary rat neuron/glia culture," Neurochemical Research, vol. 33, no. 10, pp. 2044-2053, 2008.

[103] L. Qian, H. -M. Wu, S. -H. Chen et al., " $\beta 2$-adrenergic receptor activation prevents rodent dopaminergic neurotoxicity by inhibiting microglia via a novel signaling pathway," Journal of Immunology, vol. 186, no. 7, pp. 4443-4454, 2011.

[104] A. Ghosh, A. Roy, X. Liu et al., "Selective inhibition of NF- $\kappa$ B activation prevents dopaminergic neuronal loss in a mouse model of Parkinson's disease," Proceedings of the National Academy of Sciences of the United States of America, vol. 104, no. 47, pp. 18754-18759, 2007.

[105] F. Zhang, L. Qian, P. M. Flood, J. S. Shi, J. S. Hong, and H. M. Gao, "Inhibition of $\mathrm{I} \kappa \mathrm{B}$ kinase- $\beta$ protects dopamine neurons against lipopolysaccharide-induced neurotoxicity," Journal of Pharmacology and Experimental Therapeutics, vol. 333, no. 3, pp. 822-833, 2010. 


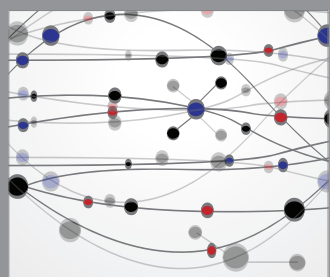

The Scientific World Journal
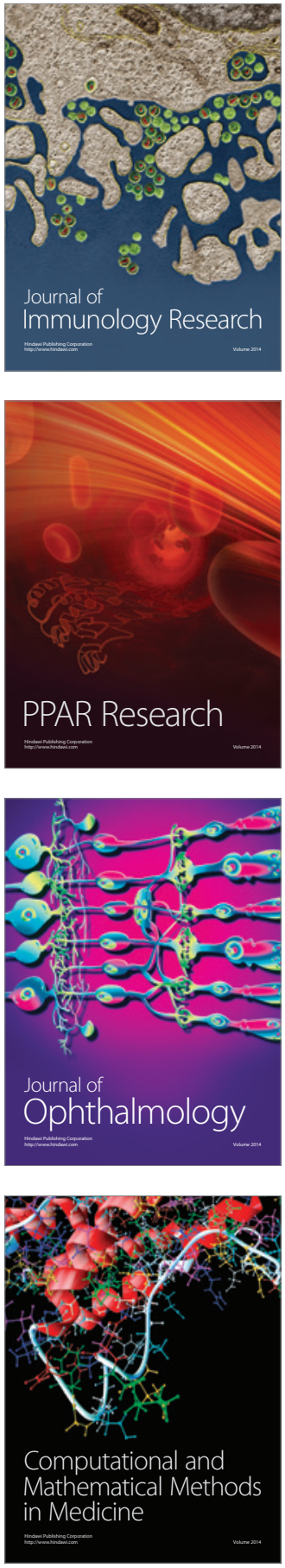

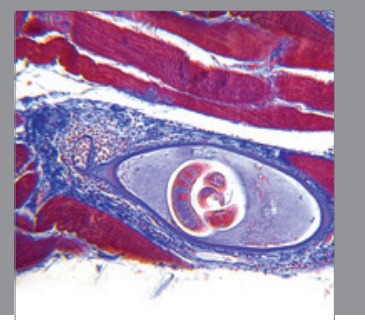

Gastroenterology

Research and Practice
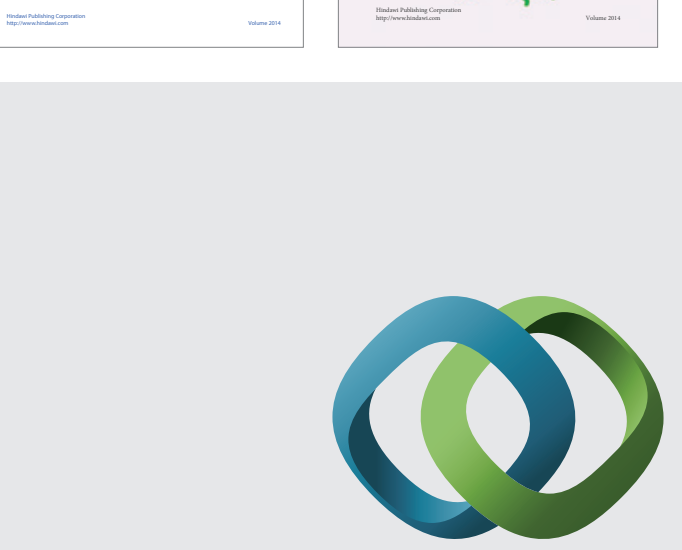

\section{Hindawi}

Submit your manuscripts at

http://www.hindawi.com
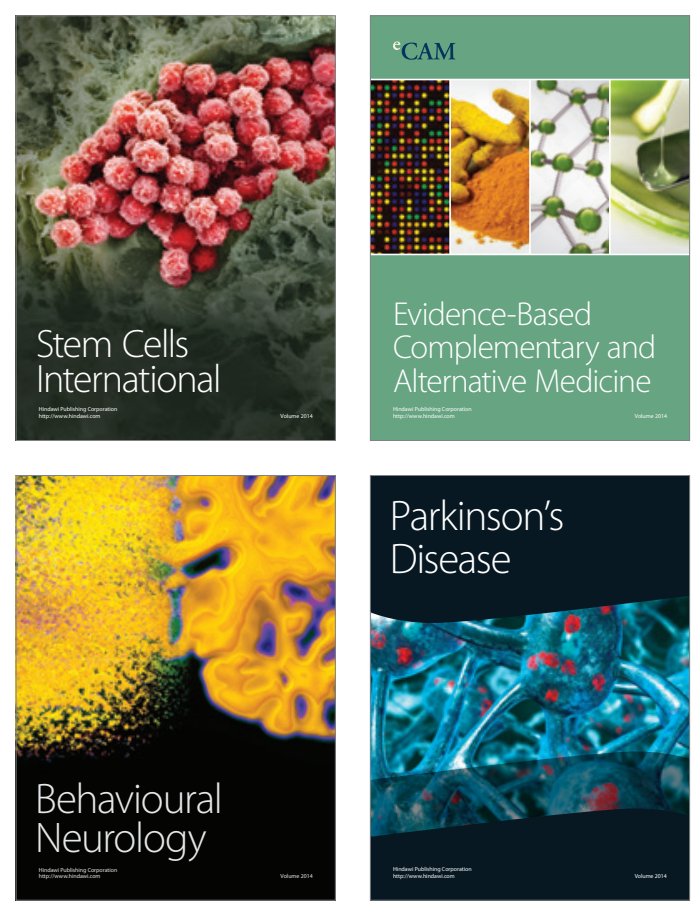

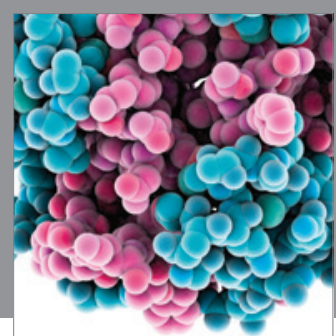

Journal of
Diabetes Research

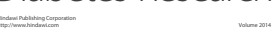

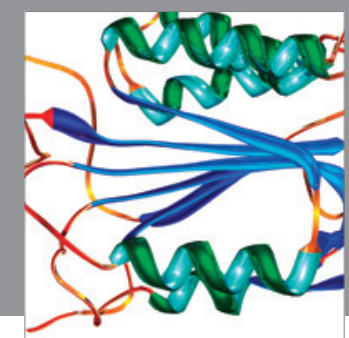

Disease Markers
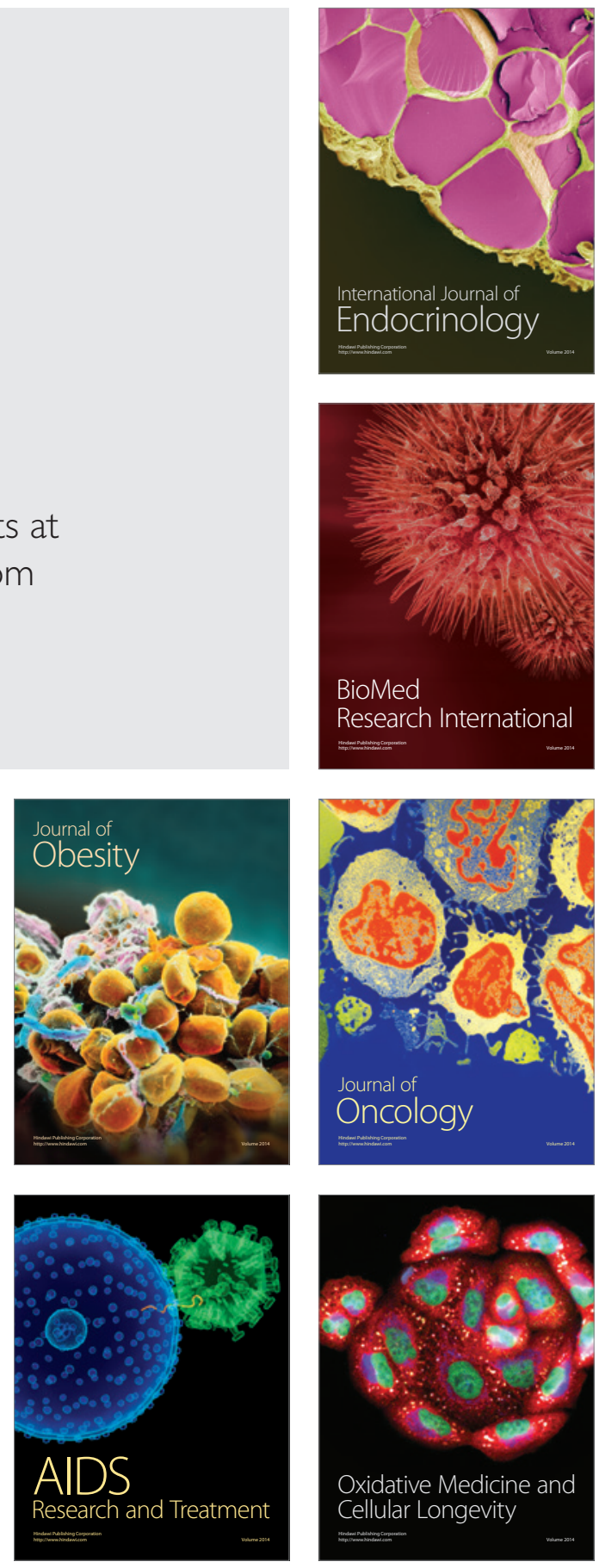